\title{
Constrained exchangeable partitions
}

\author{
Alexander Gnedin听 \\ ${ }^{1}$ Mathematical Institute, Utrecht University, P.O. Box 80010, 3508 TA Utrecht, The Netherlands
}

For a class of random partitions of an infinite set a de Finetti-type representation is derived, and in one special case a central limit theorem for the number of blocks is shown.

Keywords: exchangeability, paintbox, stick-breaking

\section{Introduction}

Under a partition of the set $\mathbb{N}$ we shall mean a sequence $\left(b_{1}, b_{2}, \ldots\right)$ of subsets of $\mathbb{N}$ such that (i) the sets $b_{j}$ are disjoint, (ii) $\cup_{j} b_{j}=\mathbb{N}$, (iii) if $b_{k}=\varnothing$ then also $b_{k+1}=\varnothing$ and (iv) if $b_{k+1} \neq \varnothing$ then $\min b_{k}<\min b_{k+1}$. Condition (iv) says that the sequence of minimal elements of the blocks is increasing. One can think of partition as a mapping which sends a generic element $j \in \mathbb{N}$ to one of the infinitely many blocks, in such a way that conditions (iii) and (iv) are fulfilled.

A random partition $\Pi=\left(B_{k}\right)$ of $\mathbb{N}$ (so, with random blocks $B_{k}$ ) is a random variable with values in the set of partitions of $\mathbb{N}$. This concept can be made precise by means of a projective limit construction and the measure extension theorem. To this end, one identifies $\Pi$ with consistent partitions $\Pi_{n}:=\left.\Pi\right|_{[n]}$ $(n=1,2, \ldots)$ of finite sets $[n]:=\{1, \ldots, n\}$. Note that the restriction $\Pi_{n}$, which is obtained by removing all elements not in $[n]$, still has the blocks in the order of increase of their least elements.

There is a well developed theory of exchangeable partitions $\left[1 ; 13,17\right.$. Recall that $\Pi=\left(B_{j}\right)$ is exchangeable if the law of $\Pi$ is invariant under all bijections $\sigma: \mathbb{N} \rightarrow \mathbb{N}$. Partitions with weaker symmetry properties have also been studied. Pitman [16] introduced partially exchangeable random partitions of $\mathbb{N}$ with the property that the law of $\Pi$ is invariant under all bijections $\sigma: \mathbb{N} \rightarrow \mathbb{N}$ that preserve the order of blocks, meaning that the sequence of the least elements of the sets $\sigma\left(B_{1}\right), \sigma\left(B_{2}\right), \ldots$ is also increasing. Pitman [16] derived a de Finetti-type representation for partially exchangeable partitions and established a criterion for their exchangeability. Kerov [14] studied a closely related structure of virtual permutations of $\mathbb{N}$, which may be seen as partially exchangeable partitions with some total ordering of elements within each of the blocks. Kallenberg [13] characterised spreadable partitions whose law is invariant under increasing injections $\sigma: \mathbb{N} \rightarrow \mathbb{N}$.

In this note we consider constrained random partitions of $\mathbb{N}$ which satisfy the condition that, for a fixed integer sequence $\rho=\left(\rho_{1}, \rho_{2}, \ldots\right)$ with $\rho_{k} \geq 1$, each block $B_{k}$ contains $\rho_{k}$ least elements of $\cup_{j \geq k} B_{j}$, for every $k$ with $B_{k} \neq \varnothing$. It is easy to check that this condition holds if and anly if the sequence comprised of $\rho_{1}$ least elements of $B_{1}$, followed by $\rho_{2}$ least elements of $B_{2}$ and so on, is itself an increasing sequence. We shall focus on the constrained partitions with the following symmetry property.

Definition 1 For a given sequence $\rho$, we call $\Pi$ constrained exchangeable if $\Pi$ is a constrained partition with respect to $\rho$ and the law of $\Pi$ is invariant under all bijections $\sigma: \mathbb{N} \rightarrow \mathbb{N}$ that preserve this property.

Since the law of $\Pi$ is uniquely determined by the laws of finite restrictions $\Pi_{n}$, the constrained exchangeability of $\Pi$ amounts to the analogous property of $\Pi_{n}$ 's for each $n=1,2, \ldots$. To gain a feeling of the property, the reader is suggested to check that for $\rho=(1,2,1, \ldots)$ the partition $\Pi_{8}$ assumes the values $(\{1,3,5\},\{2,4,6\},\{7,8\})$ and $(\{1,2,3\},\{4,5,8\},\{6,7\})$ with the same probability.

Every partition of $\mathbb{N}$ is constrained with respect to $\rho=(1,1, \ldots)$, and every constrained exchangeable partition with this $\rho$ is partially exchangeable in the sense of Pitman [16]. In principle, any constrained exchangeable partition may be reduced to some Pitman's partially exchangeable partition by isolating $\rho_{k}-1$ least elements of $B_{k}$ in $\rho_{k}-1$ singleton blocks, for each $\rho_{k}>1$, but this viewpoint will not be adopted here.

For general $\rho \neq(1,1, \ldots)$ the constrained exchangeable partitions which are also exchangeable are rather uninteresting, since they cannot have infinitely many blocks:

\footnotetext{
†gnedin@math.uu.nl
} 
Proposition 2 Let $\Pi$ be a constrained partition with respect to some $\rho$ which has $\rho_{k}>1$ for some $k$. If $\Pi$ is exchangeable then $\Pi$ has at most $k$ nonempty blocks.

Proof: Suppose $B_{k} \neq \varnothing$, then by Kingman's representation of exchangeable partitions [17] the set $\cup_{j \geq k} B_{j}$ contains infinitely many elements. For the same reason $\# B_{k} \geq 2$ implies that $B_{k}$ is an infinite set, and that partition $\Pi^{\prime}$ obtained by restricting $\Pi$ to $\cup_{j \geq k} B_{j}$ and re-labelling the elements of $\cup_{j \geq k} B_{j}$ by $\mathbb{N}$ in increasing order is an exchangeable partition of $\mathbb{N}$. But then with probability one $\Pi^{\prime}$ is the trivial singleblock partition, because elements 1 and 2 are always in the same block.

In many contexts where random partitions appear, exchangeability is an obvious kind of symmetry. Constrained exchangeability may appear when some initial elements of the blocks play a special role of 'establishing' the block. To illustrate, consider the following situation. Suppose there is a sequence of independent random points sampled from some distribution on $\mathbb{R}^{d}$. Define $D_{1}$ as the convex hull of the first $\rho_{1}$ points, $D_{2}$ as the convex hull of the first $\rho_{2}$ points not in $D_{1}, D_{3}$ as the convex hull of the first $\rho_{3}$ points not in $D_{1} \cup D_{2}$, etc. Divide $\mathbb{R}^{d}$ in disjoint nonempty subsets $G_{1}=D_{1}, G_{2}=D_{2} \backslash D_{1}, G_{3}=D_{3} \backslash\left(D_{1} \cup D_{2}\right), \ldots$. A constrained exchangeable partition $\Pi$ of $\mathbb{N}$ is defined then by assigning to block $B_{k}$ the indices of $\rho_{k}$ initial points that determine $D_{k}$ and the indices of all further points that hit $G_{k}$.

Of course, there is nothing special in the convex hulls construction, and any other way of 'spanning' a spatial domain $D_{k}$ on $\rho_{k}$ sample points and then 'peeling' the space in $G_{k}$ 's will also result in a constrained exchangeable partition. An example of this kind related to multidimensional records will be given.

In what follows we extend Pitman's [16] sequential realisation of partitions via frequencies of blocks, to cover arbitrary constrained exchangeable partitions. Generalising a result on exchangeable partitions [6] we shall also derive a central limit theorem for the number of blocks of finite partitions $\Pi_{n}=\left.\Pi\right|_{[n]}$ in one important case of partitions induced by a 'stick-breaking' scheme.

\section{Constrained sampling}

We fix throughout a sequence of positive integers $\rho$. Recall that a composition is a finite sequence of positive integers called parts, e.g. $(3,1,2)$ is a composition of $6=3+1+2$ with three parts. We say that a composition $\lambda=\left(\lambda_{1}, \ldots, \lambda_{\ell}\right)$ is a constrained composition of $n$ if $\lambda_{j} \geq \rho_{j}$ for $j=1, \ldots, \ell-1$ and $|\lambda|:=\sum \lambda_{j}=n$.

For each $\lambda$ a constrained composition of $n$, the following random algorithm, which may be called constrained sampling, yields another constrained composition $\mu$ of $n-1$. Imagine a row of boxes labeled $1, \ldots, \ell$ and occupied by $\lambda_{1}, \ldots, \lambda_{\ell}$ white balls. Let $\Lambda_{j}:=\lambda_{j}+\ldots+\lambda_{\ell}, j \leq \ell$. At the first step, $\rho_{1}$ balls in box 1 are re-painted black and then a white ball is drawn uniformly at random from all $\Lambda_{1}-\rho_{1}$ white balls. If the ball drawn was in box 1 , the ball is deleted and the new composition is $\mu=\left(\lambda_{1}-1, \lambda_{2}, \ldots, \lambda_{\ell}\right)$, and if the ball drawn was in some other box, it is returned to the box and the process continues, so that at the second step $\rho_{2}$ balls in box 2 are re-painted black and a white ball is drawn uniformly at random from boxes $2, \ldots, \ell$. If the second ball drawn was in box 2 , the ball is deleted and the new composition is $\mu=\left(\lambda_{1}, \lambda_{2}-1, \lambda_{3}, \ldots, \lambda_{\ell}\right)$, and so on. If the procedure does not terminate in $\ell-1$ steps, then a ball is deleted from the last box and the new composition is $\mu=\left(\lambda_{1}, \ldots, \lambda_{\ell-1}, \lambda_{\ell}-1\right)$. By this description, for $j<\ell$ the transition probability from $\lambda$ to $\mu=\left(\ldots, \lambda_{j}-1, \ldots\right)$ is

$$
\frac{\Lambda_{2}}{\left(\Lambda_{1}-\rho_{1}\right)} \cdots \frac{\Lambda_{j}}{\left(\Lambda_{j-1}-\rho_{j-1}\right)} \frac{\left(\lambda_{j}-\rho_{j}\right)}{\left(\Lambda_{j}-\rho_{j}\right)},
$$

while the transition probability from $\lambda$ to $\mu=\left(\cdots, \lambda_{\ell}-1\right)$ is

$$
\frac{\Lambda_{2}}{\left(\Lambda_{1}-\rho_{1}\right)} \cdots \frac{\Lambda_{\ell}}{\left(\Lambda_{\ell-1}-\rho_{\ell-1}\right)} .
$$

A random sequence $\mathcal{C}=\left(\mathcal{C}_{n}\right)$ of constrained compositions of integers $n=1,2, \ldots$ is called consistent if $\mathcal{C}_{n-1}$ has the same law as the composition derived from $\mathcal{C}_{n}$ by the above constrained sampling procedure, for each $n>1$. Every consistent sequence $\left(\mathcal{C}_{n}\right)$ is an inverse Markov chain with some co-transition probabilities depending only on $\rho$. By analogy with [5] a consistent sequence $\mathcal{C}$ will be called a constrained composition structure.

If the constraints are determined by $\rho=(1,1, \ldots)$, the constrained sampling amounts to a co-transition rule related to the partially exchangeable partitions in [16]. The unconstrained sampling (corresponding to $\rho=(0,0, \ldots))$ leads to composition structures studied in [5, 9; 10$]$. 


\section{Basic representation}

For $\Pi$ a constrained partition of $\mathbb{N}$ with blocks $B_{1}, B_{2}, \ldots$ we define, for each $n=1,2$, ., a composition $\mathcal{C}_{n}$ of $n$ as the finite sequence of positive values in $\#\left(B_{1} \cap[n]\right), \#\left(B_{2} \cap[n]\right), \ldots$. We call this composition the shape of $\Pi_{n}$ and write $\mathcal{C}_{n}=\operatorname{shape}\left(\Pi_{n}\right)$.

The number of constrained partitions of $[n]$ with shape $\lambda$ is equal to

$$
d(\lambda):=\prod_{j=1}^{\ell-1}\left(\begin{array}{c}
\Lambda_{j}-\rho_{j} \\
\lambda_{j}-\rho_{j}
\end{array}\right)
$$

Similarly, the number of partitions of $[n]$ with shape $\lambda=\left(\lambda_{1}, \ldots, \lambda_{\ell}\right)$ and whose restriction on $\left[n^{\prime}\right]$ (for $\left.n^{\prime}<n\right)$ has shape $\mu=\left(\mu_{1}, \ldots, \mu_{k}\right)$ is equal to

$$
d(\lambda, \mu):=\left[\prod_{j=1}^{\ell-1}\left(\begin{array}{c}
\mathrm{M}_{j}-\Lambda_{j} \\
\mu_{j}-\lambda_{j}
\end{array}\right)\right]\left(\begin{array}{c}
\mathrm{M}_{\ell}-\Lambda_{\ell}-\left(\rho_{\ell}-\lambda_{\ell}\right)_{+} \\
\mu_{\ell}-\rho_{\ell} \vee \lambda_{\ell}
\end{array}\right)\left[\prod_{j=\ell+1}^{k-1}\left(\begin{array}{c}
\mathrm{M}_{j}-\rho_{j} \\
\mu_{j}-\rho_{j}
\end{array}\right)\right],
$$

where $\mathrm{M}_{j}=\mu_{j}+\ldots+\mu_{k}, j \leq k$.

Introduce a function of compositions

$$
p(\lambda):=\mathbb{P}\left(\text { shape }\left(\Pi_{n}\right)=\lambda\right) .
$$

It is easy to check that the consistency of $\Pi_{n}$ 's with respect to restrictions implies that the $\mathcal{C}_{n}$ 's are consistent in the sense of constrained sampling, therefore appealing to Kolmogorov's measure extension theorem we have:

Proposition 3 The formula

$$
\mathbb{P}\left(\Pi_{n}=\cdot\right)=p(\operatorname{shape}(\cdot)) / d(\operatorname{shape}(\cdot))
$$

establishes a canonical homeomorphism between the distributions of constrained exchangeable partitions of $\mathbb{N}$ and constrained composition structures. Conditionally given $\mathcal{C}_{n}=\operatorname{shape}\left(\Pi_{n}\right)=\lambda$ the distribution of $\Pi_{n}$ is uniform on the set of constrained partitions of $[n]$ with shape $\lambda$.

The following basic construction modifies the one exploited in [14;16]. Let $\left(P_{1}, P_{2}, \ldots\right)$ be an arbitrary sequence of random variables satisfying $P_{k} \geq 0$ and $\sum_{k} P_{k} \leq 1$. A constrained exchangeable partition $\Pi$ directed by $\left(P_{k}\right)$ is defined as follows. Conditionally given $\left(P_{k}\right)$ the partition is obtained by successive extension of $\Pi_{n}$ to $\Pi_{n+1}$, for each $n=1,2, \ldots$, according to the rules: given $\Pi_{n}$ with shape $\left(\Pi_{n}\right)=$ $\left(\lambda_{1}, \ldots, \lambda_{\ell}\right)$, the element $n+1$

(i) joins the block $B_{j}, j<\ell$, with probability $P_{j}$,

(ii) if $\lambda_{\ell}<\rho_{\ell}$ joins the block $B_{\ell}$ with probability $1-\sum_{j=1}^{\ell-1} P_{j}$,

(iii) and if $\lambda_{\ell} \geq \rho_{\ell}$ joins the block $B_{\ell}$ with probability $P_{\ell}$ or starts the new block $B_{\ell+1}$ with probability $1-\sum_{j=1}^{\ell} P_{j}$

Explicitly, for the function $p$ of compositions we have the formula

$$
p(\lambda)=d(\lambda) \mathbb{E}\left\{\left[\prod_{j=1}^{\ell-1}\left(1-\sum_{i=1}^{j-1} P_{i}\right)^{\rho_{j}} P_{j}^{\lambda_{j}-\rho_{j}}\right]\left(1-\sum_{i=1}^{\ell-1} P_{i}\right)^{\rho_{\ell} \wedge \lambda_{\ell}} P_{\ell}^{\left(\lambda_{\ell}-\rho_{\ell}\right)_{+}}\right\} .
$$

The next de Finetti-type result states that the construction covers all possible constrained exchangeable partitions. The proof is only sketched, since it follows the same lines as in [14, 16].

Proposition 4 For $\Pi$ a constrained exchangeable partition, the normalised shapes shape $\left(\Pi_{n}\right) / n($ considered as sequences padded by infinitely many zeroes) converge in the product topology with probability one to some random limit $\left(P_{1}, P_{2}, \ldots\right)$ satisfying $P_{j} \geq 0$ and $\sum_{j} P_{j} \leq 1$. Conditionally given $\left(P_{k}\right)$ the partition $\Pi$ is recovered according to the above rules (i)-(iii).

Proof: The key point is to show the existence of frequencies. This can be concluded from de Finetti's theorem for $0-1$ exchangeable sequences by noting that the indicators 1 ( $m$ belongs to block $\left.B_{k}\right)$ for $m>n$ are conditionally exchangeable given that the block $B_{k}$ has at least $\rho_{k}$ representatives in $[n]$. Alternatively, one can use, as in [14], more direct Martin boundary arguments which exploit the explicit formulas (1] and 2) to show that the pointwise limit of ratios $d(\cdot, \mu) / d(\mu)$, as $m=|\mu| \rightarrow \infty$, exists if and only if $\mu_{j} / m$ converge for every $j$. 


\section{The formation sequence}

Nacu [15] established that the law of a partially exchangeable partition is uniquely determined by the law of the increasing sequence of the least elements of blocks. We show that a similar result holds for every constrained exchangeable partition $\Pi$ with general $\rho$.

We define the formation sequence to be the sequence obtained by selecting the $\rho_{k}$ th least element of the block $B_{k}$ of $\Pi$, for $k=1,2, \ldots$. For a composition $\lambda$ let $q(\lambda)$ be the probability that the formation sequence starts with elements $\lambda_{1}, \lambda_{1}+\lambda_{2}, \ldots, \lambda_{1}+\ldots+\lambda_{\ell}$. Let $\left(P_{j}\right)$ be the frequencies as in Section 3 . and introduce the variables

$$
H_{k}=1-\sum_{j=1}^{k} P_{j}, \text { so } P_{k}=H_{k-1}-H_{k}
$$

where we set $H_{0}=1$. Then

$$
q\left(\lambda_{1}, \ldots, \lambda_{\ell}\right)=\mathbb{E}\left[\prod_{j=1}^{\ell-1}\left(\begin{array}{c}
\lambda_{j+1}-1 \\
\rho_{j+1}-1
\end{array}\right) H_{j}^{\rho_{j+1}}\left(1-H_{j}\right)^{\lambda_{j+1}-\rho_{j+1}}\right] .
$$

Comparing this with (3) written in the same variables (where $H_{0}=1$ ) we obtain for constrained compositions

$$
p(\lambda)=d(\lambda) \mathbb{E}\left\{\left[\prod_{j=1}^{\ell-1} H_{j-1}^{\rho_{j}}\left(H_{j-1}-H_{j}\right)^{\lambda_{j}-\rho_{j}}\right] H_{\ell-1}^{\rho_{\ell} \wedge \lambda_{\ell}}\left(H_{\ell-1}-H_{\ell}\right)^{\left(\lambda_{\ell}-\rho_{\ell}\right)_{+}}\right\}
$$

which leads to the following conclusion:

Proposition 5 There is an invertible linear transition from $p$ to $q$. Hence each of these two functions on compositions uniquely determines the law of $\Pi$.

Proof: The substantial part of the claim is showing that we can compute $p$ from $q$. To that end, start by observing that $p$ is uniquely determined by the values on compositions of the type $\left(\lambda_{1}, \ldots, \lambda_{\ell-1}, \rho_{\ell}\right)$. To see that this follows from the consistency for various $n$, argue by induction in $m=0, \ldots, \rho_{\ell}$ for compositions $\left(\ldots, \rho_{\ell}-m\right)$. Now, for such compositions whose last part meets the constraint exactly, (5) and 4 involve the same factors of the type $H_{j}^{\rho_{j}}$, hence $p$ can be reduced to $q$ by expanding each factor $\left(H_{j-1}-H_{j}\right)^{k}=\left(\left(1-H_{j}\right)-\left(1-H_{j-1}\right)\right)^{k}$ using the binomial formula.

\section{The paintbox}

Paintbox representations based on the uniform sampling from $[0,1]$ are often used to model exchangeable structures and their relatives [5; 8; 9; 17]. We shall design a version that is appropriate for constrained exchangeable partitions.

Let $1=H_{0} \geq H_{1} \geq H_{2} \geq \ldots \geq 0$ be an arbitrary nonincreasing random sequence. Let $\left(U_{n}\right)$ be a sequence of independent $[0,1]$-uniform random points, independent of $\left(H_{k}\right)$. We define a new sequence $\left.\left(U_{n}\right)\right|_{\rho}\left(H_{k}\right)$ with some of $U_{n}$ 's replaced by $H_{k}$ 's, as follows. Replace $U_{1}, \ldots, U_{\rho_{1}}$, by $H_{1}$. Then replace the first $\rho_{2}$ entries which belong to $U_{\rho_{1}}, U_{\rho_{1}+1}, \ldots$ and hit $\left[0, H_{1}\right.$ [ by $H_{2}$. Inductively, when $H_{1}, \ldots, H_{k}$ get used, respectively, $\rho_{1}, \ldots, \rho_{k}$ times, keep on screening uniforms until replacing the first $\rho_{k+1}$ points hitting $\left[0, H_{k}\right.$ [ by $H_{k+1}$. Eventually all $H_{k}$ 's will enter the resulting sequence.

The construction has an interpretation in terms of the classical theory of records (see [7; 14] for a special case).

Proposition 6 Conditionally given $\left(H_{k}\right)$, the sequence $\left.\left(U_{n}\right)\right|_{\rho}\left(H_{k}\right)$ has the same distribution as $\left(U_{n}\right)$ conditioned on the event that the sequence of lower records in $\left(U_{n}\right)$ is $\left(H_{k}\right)$, with the record value $H_{k}$ repeated $\rho_{k}$ times.

In this framework, we define a partition $\Pi$ by assigning to block $B_{k}$ all integers which label the entries of $\left.\left(U_{n}\right)\right|_{\rho}\left(H_{k}\right)$ falling in $\left[H_{k}, H_{k-1}\left[\right.\right.$. Given $\left(H_{k}\right)$, the chance for $U_{n}$ to hit $\left[H_{j}, H_{j-1}\left[\right.\right.$ is $P_{j}=H_{j-1}-H_{j}$, therefore the construction is equivalent to that defined by the rules (i)-(iii) above. 


\section{Stick-breaking partitions}

Explicit evaluation of the function $p$ is possible when the frequencies involve a kind of independence. To this end, it is convenient to introduce yet another set of variables $\left(W_{k}\right)$ (sometimes called residual fractions) which satisfy $H_{k}=W_{1} \cdots W_{k}, W_{k} \in[0,1]$. In these variables $(5)$ becomes

$$
p(\lambda)=d(\lambda) \mathbb{E}\left\{\left[\prod_{j=1}^{\ell-1} W_{j}^{\Lambda_{j}-\lambda_{j}}\left(1-W_{j}\right)^{\lambda_{j}-\rho_{j}}\right]\left(1-W_{\ell}\right)^{\left(\lambda_{\ell}-\rho_{\ell}\right)_{+}}\right\}
$$

where $\Lambda_{j}=\lambda_{j}+\ldots+\lambda_{\ell}$. As in [16], if the $W_{k}$ 's are independent, [6] assumes the product form

$$
p(\lambda)=\prod_{k=1}^{\ell} q_{k}\left(\Lambda_{k}: \lambda_{k}\right)
$$

with the decrement matrices

$$
q_{k}(n: m)=\left(\begin{array}{c}
n-\rho_{k} \\
m-\rho_{k}
\end{array}\right) \mathbb{E}\left[\left(1-W_{k}\right)^{m-\rho_{k}} W_{k}^{n-m}\right], \quad 1 \leq m \leq n,
$$

and the convention $\left(\begin{array}{c}-i \\ -j\end{array}\right)=1(i=j)$ for negative arguments of the binomial coefficients. In fact, 77 forces representation (8) (this fact is implicit in [14; 16] in the case of partially exchangeable partitions):

Proposition 7 A constrained composition structure $\left(\mathcal{C}_{n}\right)$ satisfies (7) with some decrement matrices $q_{k}, k=1,2, \ldots$, if and only if there exist independent $[0,1]$-valued random variables $\left(W_{k}\right)$ such that (8) holds.

Proof: We argue the 'only if' part. For $n<\rho_{1}$ we have $q_{1}(n: n)=1$ by definition. For $n \geq \rho_{1}$ the constrained sampling consistency yields

$$
q_{1}(n: m)=\frac{m+1-\rho_{1}}{n+1-\rho_{1}} q_{1}(n+1: m+1)+\frac{n+1-m}{n+1-\rho_{1}} q_{1}(n+1: m)
$$

which is the familiar Pascal-triangle recursion in the variables $n-\rho_{1}, m-\rho_{1}$, therefore the integral representation (8) follows as a known consequence of the Hausdorff moments problem. The case $k>1$ is completely analogous. The independence of the $W_{k}$ 's is obvious from (7).

We note in passing that the product formula (7) with a single decrement matrix leads, in a related setting of regenerative composition structures, to a nonlinear recursion and a very different conclusion [9]. See [11] for product formulas of another kind in the exchangeable case.

Suppose now that $W_{k}$ 's are independent and have beta $\left(a_{k}, b_{k}\right)$ distributions, whose density is

$$
(1-s)^{a_{k}-1} s^{b_{k}-1} / \mathrm{B}\left(a_{k}, b_{k}\right) .
$$

The rows of the decrement matrices are then Pólya-Eggenberger distributions

$$
q_{k}(n: m)=\left(\begin{array}{c}
n-\rho_{k} \\
m-\rho_{k}
\end{array}\right) \frac{\left(a_{k}\right)_{m-\rho_{k}}\left(b_{k}\right)_{n-m}}{\left(a_{k}+b_{k}\right)_{n-\rho_{k}}}, \quad m=1, \ldots, n .
$$

For instance, taking positive integer $a_{k}, b_{k}$ and $\rho_{k}=a_{k}+b_{k}-1$, a partition $\Pi$ is constructed as follows: replace $U_{1}, \ldots, U_{\rho_{1}}$ by the value $H_{1}$ equal to the $b_{1}$ th minimal order statistic of these points, then replace the first $\rho_{2}$ uniforms that hit $\left[0, H_{1}\right.$ [ by the value $H_{2}$ equal to the $b_{2}$ th minimal order statistic of these hits, etc, thus defining a partition via $\left.\left(U_{n}\right)\right|_{\rho}\left(H_{k}\right)$. A distinguished class of structures of this kind is the EwensPitman two-parameter family of exchangeable partitions [12; 16; 17] with $\rho=(1,1, \ldots), a_{k}=\theta+k \alpha$ and $b_{k}=1-\alpha$ (for suitable $\alpha$ and $\theta$ ); the product formula simpifies in this case due to a major telescoping of factors.

\section{Counting the blocks}

Let $K_{n}$ be the number of blocks of $\Pi_{n}$, which in the $\left(U_{j}\right) \mid \rho\left(H_{k}\right)$-representation coincides with the number of intervals $\left.\left.\left.] H_{1}, H_{0}\right],\right] H_{2}, H_{1}\right], \ldots$ discovered by the $n$ first terms of the sequence. Conditionally given $\left(H_{k}\right), K_{n}$ is the number of certain independent geometric summands which sum to no more than $n$. In 
particular, the difference between the $k$ th and the $(k+1)$ st entries of the formation sequence follows the negative binomial distribution with parameters $\rho_{k}, H_{k}$.

We shall proceed by assuming a 'stick-breaking' scheme $H_{k}=W_{1} \cdots W_{k}, k=1,2, \ldots$, with independent identically distributed $W_{k}$ 's. We assume further that the logarithmic moments $\mu=\mathbb{E}\left[-\log W_{1}\right]$, $\sigma^{2}=\operatorname{Var}\left[-\log W_{1}\right]$ are both finite. The idea is to derive a CLT for $K_{n}$ from the standard CLT for renewal processes [4]. Similar technique was used in [6, 7], but in the new stuation we need to also limit the growth of $\rho_{k}$ as $k \rightarrow \infty$.

We will show that $K_{n}$ is asymptotic to $J_{n}:=\max \left\{k: H_{k}>1 / n\right\}$. The last quantity is indeed asymptotically Gaussian with the mean $(\log n) / \mu$ and the variance $(\log n) /\left(\sigma^{2} \mu^{-3}\right)$ because $J_{n}$ is just the number of renewal epochs within $[0, \log n]$ of the renewal process with steps $-\log W_{k}$. In loose terms, we will exploit a 'cut-off phenomenon': typically, only a few points out of $n$ uniforms fall below $1 / n$, while for $k<J_{n}$ essentially all intervals get hit, with exponentially growing occupancy numbers when scanned backwards in $k$ from $k=J_{n}$.

Proposition 8 Suppose $\Pi$ is directed by $H_{k}=W_{1} \cdots W_{k}$, where for $k=1,2, \ldots$ the $W_{k}$ 's are i.i.d. with finite logarithmic moments $\mu=\mathbb{E}\left[-\log W_{1}\right], \sigma^{2}=\operatorname{Var}\left[-\log W_{1}\right]$. If

$$
\log \left[\sum_{j=1}^{k} \rho_{j}\right]=o(k), \text { as } k \rightarrow \infty,
$$

then the strong law of large numbers holds, i.e. $K_{n} \sim \mu^{-1} \log n$ a.s.. Moreover, the random variable $\left(K_{n}-\mathbb{E}\left[K_{n}\right]\right) / \sqrt{\operatorname{Var}\left[K_{n}\right]}$ converges in law to the standard Gaussian distribution, whereas the moments satisfy

$$
\mathbb{E}\left[K_{n}\right] \sim \frac{\log n}{\mu}, \quad \operatorname{Var}\left[K_{n}\right] \sim \frac{\log n}{\sigma^{2} \mu^{-3}} .
$$

Proof: By the construction of $\left.\left(U_{j}\right)\right|_{\rho}\left(H_{k}\right)$, we have a dichotomy: $\left.\left.U_{n} \in\right] H_{k}, H_{k-1}\right]$ implies that either $U_{n}$ will enter the transformed sequence or will get replaced by some $H_{i} \geq H_{k}$. Let $U_{1 n}<\ldots<U_{n n}$ be the order statistics of $U_{1}, \ldots, U_{n}$. It follows that

(i) if $U_{j n}>H_{k}$ then $K_{n} \leq j+k$,

(ii) if $U_{m n}<H_{k}$ for $m=\sum_{i=1}^{k} \rho_{k}$ then $K_{n} \geq k$.

Define $\xi_{n}$ by $U_{\xi_{n}, n}<1 / n<U_{\xi_{n+1}, n}$ and recall that $J_{n}$ was defined by $H_{J_{n}+1} \leq 1 / n<H_{J_{n}}$, thus $\xi_{n}$ is the number of uniforms to the left of $1 / n$, and $J_{n}$ follows the CLT. Clearly, $J_{n}$ and $\xi_{n}$ are independent and $\xi_{n}$ is binomial $(n, 1 / n)$. By (i), we have $K_{n} \leq J_{n}+\xi_{n}$ where $\xi_{n}$ is approximately Poisson(1), which yields the desired upper bound.

The lower bound is more delicate. Introduce

$$
\psi_{n}=c \sum_{j=1}^{\lfloor\log n\rfloor} \rho_{j}
$$

where $c$ should be selected sufficiently large. Then by the assumption $99 \log \psi_{n}=o(\log n)$, which is enough to assure that the number, say $L_{n}$, of the $H_{k}$ 's larger than $\psi_{n} / n$ is still asymptotic to $J_{n}$. Because $L_{n}$ is close to Gaussian with moments as in (10), an easy large deviation estimate implies that the inequality $L_{n}<(c / 2) \log n$ holds with probability at least $1-n^{-2}$. On the other hand, the number of uniforms smaller $\phi_{n} / n$ is also close to Gaussian with both central moments about $\phi_{n}$, hence, in view of $\phi_{n}>c \log n$, a similar estimate shows that this number is at least $(c / 2) \log n$ with probability at least $1-n^{-2}$. By application of (ii) with $k=L_{n}$ we see that the lower bound $K_{n}>L_{n}$ holds up to an event of probability $O\left(n^{-2}\right)$. This completes the proof of the CLT. Finally, since both $J_{n}$ and $L_{n}$ are asymptotic to $\mu^{-1} \log n$ almost surely, the Borel-Cantelli lemma implies that the same is valid for $K_{n}$.

\section{A continuous time process}

The sequential construction of $\Pi$ from the frequencies $\left(P_{k}\right)$ can be embedded in continuous time by letting the elements $1,2, \ldots$ arrive at epochs of a rate- 1 Poisson process on $\mathbb{R}_{+}$. Let $R_{t}$ be the total frequency of 
the blocks which are not represented by the elements arrived before $t$, then $R=\left(R_{t}\right)$ is a nonincreasing pure-jump process with piecewise-constant paths and $R_{0}=1$.

Suppose as in Section 7 that $W_{k}$ 's are independent and identically distributed. The process $R$ is then easy to describe: if after the $(k-1)$ st jump the process $R$ is in state $s$ then the time in this state has distribution gamma $\left(\rho_{k}, s\right)$, and thereafter the state is changed to $s W_{k}$. The sojourns in consequtive states $1, W_{1}, W_{1} W_{2}, \ldots$ are independent. The instance $\rho=(1,1, \ldots)$ corresponds to a known self-similar Markov process which appears as a 'tagged particle' process in random fragmentation models [1]. For general $\rho$ the process is no longer Markovian, as one needs to also include the time spent in the current state to summarise the history.

A minor adjustment of Proposition 8 to the continuous-time setting allows to conclude that under the same assumptions the number of jumps during the time $[0, T]$ is approximately Gaussian, as $T \rightarrow \infty$. In fact, the process $R$ is well defined for arbitrary positive values $\rho_{k}(k=1,2, \ldots)$, in which case an analogous CLT is readily acquired by interpolation from the case of integer $\rho_{k}$ 's.

\section{Example: the chain records}

Next is an example of Pitman's partially exchangeable partitions, so the constraint is $\rho=(1,1, \ldots)$. Consider a Borel space $\mathcal{Z}$ endowed with a distribution $\mu$ and some measurable strict partial order $\prec$. For a sample $V_{1}, V_{2}, \ldots$ from $(\mathcal{Z}, \mu)$, we say that a chain record occurs at index $j$ if either $j=1$, or $j>1$ and $V_{j}$ is $\prec$-smaller than the last chain record in the sequence $V_{1}, \ldots, V_{j-1}$. The instance of $\mathbb{R}^{d}$ with the natural coordinate-wise partial order was discussed in [7].

Let $R_{k}, k=1,2, \ldots$, be the sample values when the chain records occur; the sequence $\left(R_{k}\right)$ is a 'greedy' falling chain of the partially ordered sample $\left(V_{j}\right)$. Introducing the lower sets $L_{v}:=\{u \in \mathcal{Z}: u \prec v\}$, we define $D_{k}:=L_{R_{k}}, G_{k}:=D_{k} \backslash D_{k-1}$ (where $D_{0}:=\varnothing$ ), and we define a constrained exchangeable partition $\Pi=\left(B_{k}\right)$ as in Section 1 The frequencies of $B_{k}$ 's are $P_{k}=\mu\left(L_{G_{k}}\right)$, and we have $H_{k}=\mu\left(L_{R_{k}}\right)$, as is easily seen.

To guarantee a 'stick-breaking' form of $\left(H_{k}\right)$, as in Section 6, we need to assume a self-similarity property of the sampling space. We may call $(\mathcal{Z}, \mu, \prec)$ regenerative if (i) $\mu\left(L_{v}\right)>0$ for $\mu$-almost all points $v \in \mathcal{Z}$, and (ii) the lower section $L_{v}$ with conditional measure $\mu(\cdot) / \mu\left(L_{v}\right)$ is isomorphic, as a partially ordered probability space, to the whole space $(\mathcal{Z}, \mu, \prec)$. Since all $L_{v}$ 's are in this sense the same, the $H_{k}$ 's undergo stick-breaking with i.i.d. residual fractions whose distribution is the same as that of $L_{V_{1}}$. Under the hypothesis of Proposition 8 , the number of chain records among the first $n$ sample points is approximately Gaussian, since this number coincides with the number of blocks of $\Pi_{n}$. A class of regenerative spaces is comprised of the Bollobás-Brightwell box-spaces [3], which have all intervals $\{u: v \prec u \prec w\}$ for $v \prec w$ isomorphic to the whole space (and not only lower sections).

Further examples of regenerative spaces appear, in a disguise, in the context of multidimensional data structures like quad-trees or simplex-trees [2]. More generally, constrained exchangeability appears in connection with data structures which allow multiple key storage at a node of the search tree.

\section{References}

[1] J. Bertoin, Random fragmentation and coagulation processes, Cambridge Univ. Press., 2006.

[2] L. Devroye, Universal limit laws for depths in random trees, SIAM J. Comp. 28 (1999) 409-432.

[3] B. Bollobás and G. Brightwell, Box spaces and random partial orders, Trans. Amer. Math. Soc. 124 (1991) 59-72.

[4] W. Feller, An introduction to probability theory and its applications, vol. 2, Wiley, NY, 1971.

[5] A. V. Gnedin, The representation of composition structures, Ann. Probab. 25 (1997) 1437-1450.

[6] A.V. Gnedin, The Bernoulli sieve, Bernoulli 10 (2004) 79-96.

[7] A.V. Gnedin, Counting the chain records: the product case, 2005 arXiv: math.PR/0510042.

[8] A.V. Gnedin and G.I. Olshanski, Coherent random permutations with descent statistic and the boundary problem for the graph of zigzag diagrams, Int. Math. Res. Notes (2006) Article ID 51968.

[9] A. V. Gnedin and J. Pitman, Regenerative composition structures, Ann. Probab. 33 (2005) 445-479. 
[10] A. V. Gnedin and J. Pitman, Regenerative partition structures, Elec. J. Comb. 11 (2005) paper R12.

[11] A. V. Gnedin and J. Pitman, Gibbs exchangeable partitions and Stirling triangles, Zapiski Nauchnych Seminarov POMI 325 (2005) 82-103.

[12] A. V. Gnedin and J. Pitman, Self-similar and Markov composition structures, Zapiski Nauchnych Seminarov POMI 326 (2005) 59-84.

[13] O. Kallenberg, Probabilistic symmetries and invariance principles, Springer, NY, 2005.

[14] S. Kerov, Subordinators and permutations action with quasi-invariant measure, Zapiski Nauchnych Seminarov POMI 223 (1995) 181-218 (translated in J. Math. Sci. (New York)).

[15] S. Nacu, Increments of random partitions, Combinatorics, Probability and Computing (2006) arXiv:math.PR/0310091

[16] J. Pitman, Exchangeable and partially exchangeable random partitions. Prob. Th. Rel. Fields 102 (1995) 145-158.

[17] J. Pitman, Combinatorial stochastic processes (Lect. Notes for 2002 St. Flour Course), Springer L. Notes Math. 2006. 